\title{
Environmental data management with the River Basin Information System
}

\author{
$\underline{\text { F. Zander }}^{\text {a }}$, S. Kralisch ${ }^{\text {a }}$, C. Busch ${ }^{\text {b }}$ and W.-A. Flügel ${ }^{\text {a }}$ \\ ${ }^{a}$ Department of Geoinformatics, Hydrology and Modelling, School of Chemical and Earth Sciences, \\ Friedrich-Schiller-University, Jena, Germany \\ ${ }^{b}$ codematix GmbH, Jena, Germany \\ Email: Franziska.Zander@uni-jena.de
}

\begin{abstract}
The River Basin Information System (RBIS), developed at the Department of Geoinformatics at the Friedrich Schiller University of Jena, provides a web-based platform for environmental data management and data sharing. It is a modular-structured, user-friendly information system that addresses the management, analysis, visualization and presentation of different types of data in the context of multidisciplinary environmental planning. RBIS is also a standalone part of the Integrated Landscape Management System (ILMS), which is a modular software integrating different steps of environmental systems analysis and planning in a flexible and user-friendly workflow.
\end{abstract}

A main focus of RBIS (see Figure 1) lies in the maintenance of metadata to ensure that information about data provenance, genesis and responsibility are not lost during processing steps applied by external tools or internal functionalities. The corresponding RBIS module stores meta-information about different data types (e.g. time series data) and offers flexible user interfaces for their visualization and manipulation.

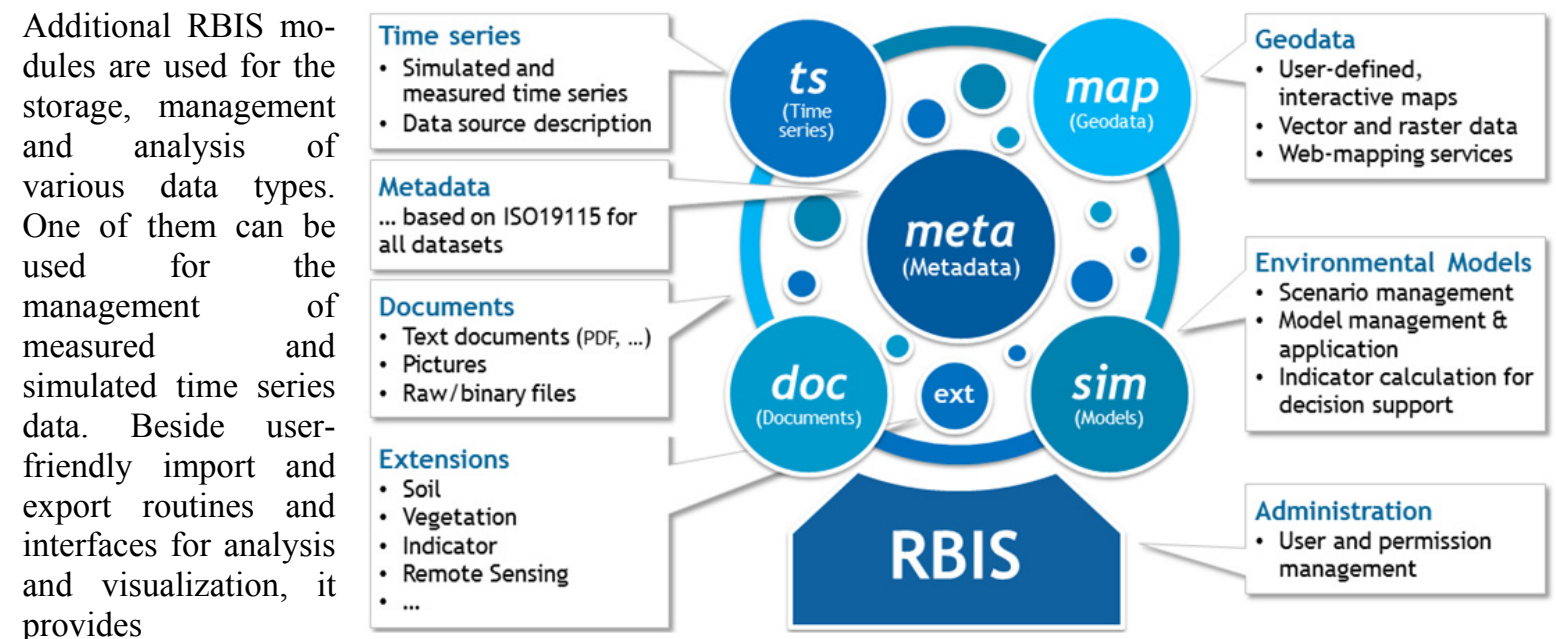

functionalities for gap

detection, rule-based

Figure 1. Layout of RBIS.

gap filling and statistical analyses. Another RBIS module allows the management and visualization of vector and gridded geo-data together with associated meta-information according to the ISO 19115 standard. Moreover, RBIS features additional modules to manage vegetation and soil measurement data, maintain input and output data for environmental simulation models and store documents. Due to a special linkage functionality, data from different RBIS modules can be associated with each other to form problem-tailored views for environmental managers and other users. An overarching part of RBIS offers functionalities related to administration, e.g. a user and permission management, dataset ownership information or logging.

In this paper we present the environmental information system RBIS as a standalone application and integrated part of ILMS for data management, analysis and exchange. The RBIS architecture will be presented as well as selected features for the management and visualization of environmental data. Further, processing and exchange functionalities in the context of the usage of RBIS in ILMS will be outlined.

Keywords: environmental information systems, integrated landscape management, hydrological modeling 


\section{INTRODUCTION}

The need for a detailed understanding of environmental systems and strategies, for their adequate management, is gaining more and more importance as we face complex problems arising from global climate change, population growth and socio-economic development. In order to create strategies for the sustainable use and management of such systems and to assess the complex interactions of their underlying processes, a variety of data coming from different sources and disciplines, e.g. remote sensing, hydrology, biology, soil science or socio-economic sciences, have to be managed and integrated. To tackle this challenge, flexible data management and information systems are needed. In order to provide meaningful information for decision makers and environmental scientists, these systems should not only support the mere storage of environmental data, but also their description with comprehensive and standardized metadata.

To address the above mentioned needs the River Basin Information System (RBIS), as a web-based platform for environmental data management and data sharing, was developed at the Department of Geoinformatics at the Friedrich Schiller University of Jena.

\section{RIVER BASIN INFORMATION SYSTEM (RBIS)}

RBIS is a modular-structured, web-based information system for the management, analysis, visualization and presentation of different types of data in the field of multidisciplinary environmental planning. The system was originally designed as a project database to provide a platform for data sharing in multidisciplinary research projects, mainly related to integrated water resource management.

The common layout of RBIS follows a 3-tier architecture. On the server side, the system is implemented using a standard Linux web stack with Apache web server, PHP programming language, PostgreSQL database management system (http://www.postgresql.org) and PostGIS extension (http://www.postgis.org) for spatial data support. As a client any current web browser can be used. In case of no or slow internet connectivity, and for an easy distribution, RBIS may also be operated in a virtual server environment using the VirtualBox (http://www.virtualbox.org) software package. The whole system is built based on open source software, ensuring a cost-efficient deployment and operation.

The following sections will provide a short overview about the system layout, introduce its time series data management, geo-data processing and other core functionalities and show examples of current RBIS implementations.

\subsection{RBIS layout}

RBIS is built in a modular way to enable a flexible use according to actual requirements. One of the few mandatory modules provides a flexible and adaptable web-based interface for the management of metadata (RBISmeta). The module is based on an implementation of the ISO 19115 Standard for Geodata (ISO 2003) and was used as the starting point for the definition of other (meta) data types. To generate different forms to view, search, edit and interlink datasets, XML-files are used as description layers (for more detail see Kralisch et al., 2009). Therefore it is very flexible and new modules can be added easily.

As shown in Figure 1 the core RBIS modules are:

- RBISts for the management, analysis, statistical processing, gap filling and visualization of measured and simulated time series data,

- RBISmap for the description and visualization of vector or raster data and for linking all types of stored geometry objects (points, polygons, layer) to metadata stored in RBIS,

- RBISdoc to store documents and their metadata (e.g. project related PDF documents) or to associate documents to other dataset in RBIS (e.g. images associated to a measurement station), and

- RBISsim to store information related to the management of simulation runs on environmental models (e.g. parameter sets) as well as indicator calculation and scenario management.

The modules RBISts and RBISmap will be explained in more detail in section 2.2 and 2.3.

In addition to these core modules various optional extensions are available. One example is the RBISsoil module for the storage of soil profile and horizon data with a special focus on hydrologically important parameters. Another one is RBISobserv, which has been developed to describe field observations (e.g. field trip, site inspection, water and soil sample) using a short description together with their spatial relation and related documents or files (e.g. pictures). For the description of future development scenarios and the management of related environmental indicators along with their values, the module RBISind provides appropriate functionalities. Here, indicator values (e.g. single values, time series data, diagrams, maps) can 
be associated with scenarios (described in the module RBISsim) and optionally to regions (e.g. a hydrological catchment or an administrative area) to describe and assess future development scenarios.

\subsection{Time series data management and processing}

One of the main modules in RBIS handles the management of time series data (RBISts). To make sure information about data provenance and genesis are not lost during data processing, RBIS provides functions for describing time series data and their processing by detailed metadata. The following steps show a typical workflow of time series handling in RBIS:

- Creation of data source location: Time series are always maintained along with their data source, i.e. a measuring station or a simulation model. As they usually have a spatial reference, they can be identified via map-based exploration and easily used in geo-statistical analyses. If available, additional information about the corresponding river system, represented area, station operator and year of establishment and closing are stored.

- Creation of time series metadata: To import new time series data it is necessary to create a new set of referring metadata. This includes information about the responsible person or organization, distributor, data description and lineage statements as well as information automatically filled and derived during data import (e.g. start and end date, time step and used default value).

- Import of time series data: In preparation for the usage in environmental models, time series are checked during the import process. This includes consistency checks regarding the format and date as well as the detection and recording of data gaps.

- Analyzing: Once time series data have been imported the user can visualize them (Figure 2), analyze them in terms of location, parameter type, unit, elevation, distance to neighboring stations or calculate their correlation to other time series data with the same parameter and unit. Since all gap information is stored as metadata it is also possible to find matching surrounded time series data to fill these data gaps.

- Fill data gaps: Gap filling can be performed by using the rule based gap filling toolbox integrated in RBIS or with the help of external tools. In both cases, the gap data are stored in RBIS while still maintaining the original data. The internal filling functions include various standard procedures (e.g. inverse distance weighting with elevation correction, linear regression, nearest neighbor or linear interpolation) and can be combined and parameterized as needed (e.g. combining methods for short and longer gaps or setting a maximum search radius for considering time series from neighboring stations). For each gap the used time series, methods and parameter sets will be stored as metadata to make sure that the result is reproducible and the genesis of the filled time series data is stored.

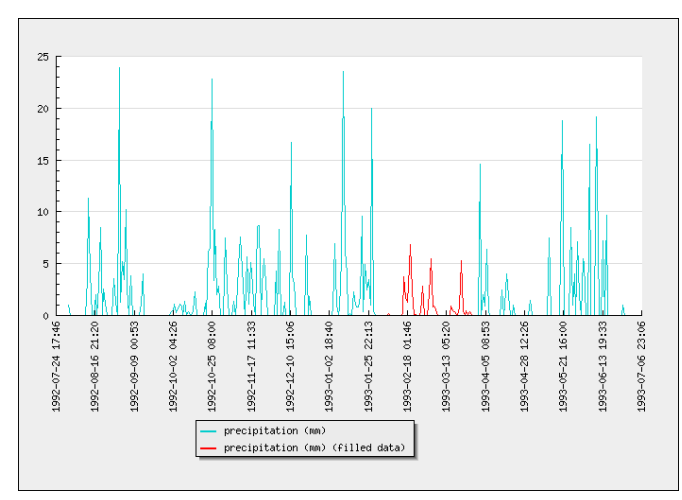

Figure 2. Time series data with filled gap.

- Export: Stored time series data can be exported and accessed in different ways:

- download the originally imported data file

- $\quad$ export the complete dataset as it is stored in the database

- $\quad$ export the time series data in a selected time intervals

- $\quad$ export the time series data with or without filled gaps

- $\quad$ export the time series data aggregated in different time steps

In addition to these export functions RBIS provides an interface to create input files for the environmental modelling framework JAMS (see section 3.2).

\subsection{Geo-data management}

In order to manage and visualize spatial data the module RBISmap was developed. RBISmap uses the $U M N$ MapServer to create maps and the OpenLayers library for a user friendly display of map data in a web browser (Figure 3). RBISmap can handle vector data (ESRI Shapefile) and raster data (GeoTIFF, JPEG).

Each uploaded dataset is described by metadata according to the ISO 19115 standard for geographic information metadata before the data can be used elsewhere (e.g. as a layer in maps). The newly created metadata dataset is automatically linked to all maps that the layer is used in. The linkage allows an easy switching between metadata and associated maps. 
Taking advantage of OpenLayers, maps are visualized in a user friendly, feature-rich frontend, including zooming/moving the map, changing the order of the displayed layers, searching for geometry objects and displaying their attribute values.

Stations and all datasets with a given location (e.g. soil, vegetation) can be automatically linked to a map and back which opens the possibility to search for datasets not only by metadata but also by their spatial location in a map. An example is the link between a measurement station and the corresponding point on a layer/map, which will be automatically created during station creation based on type and location information.

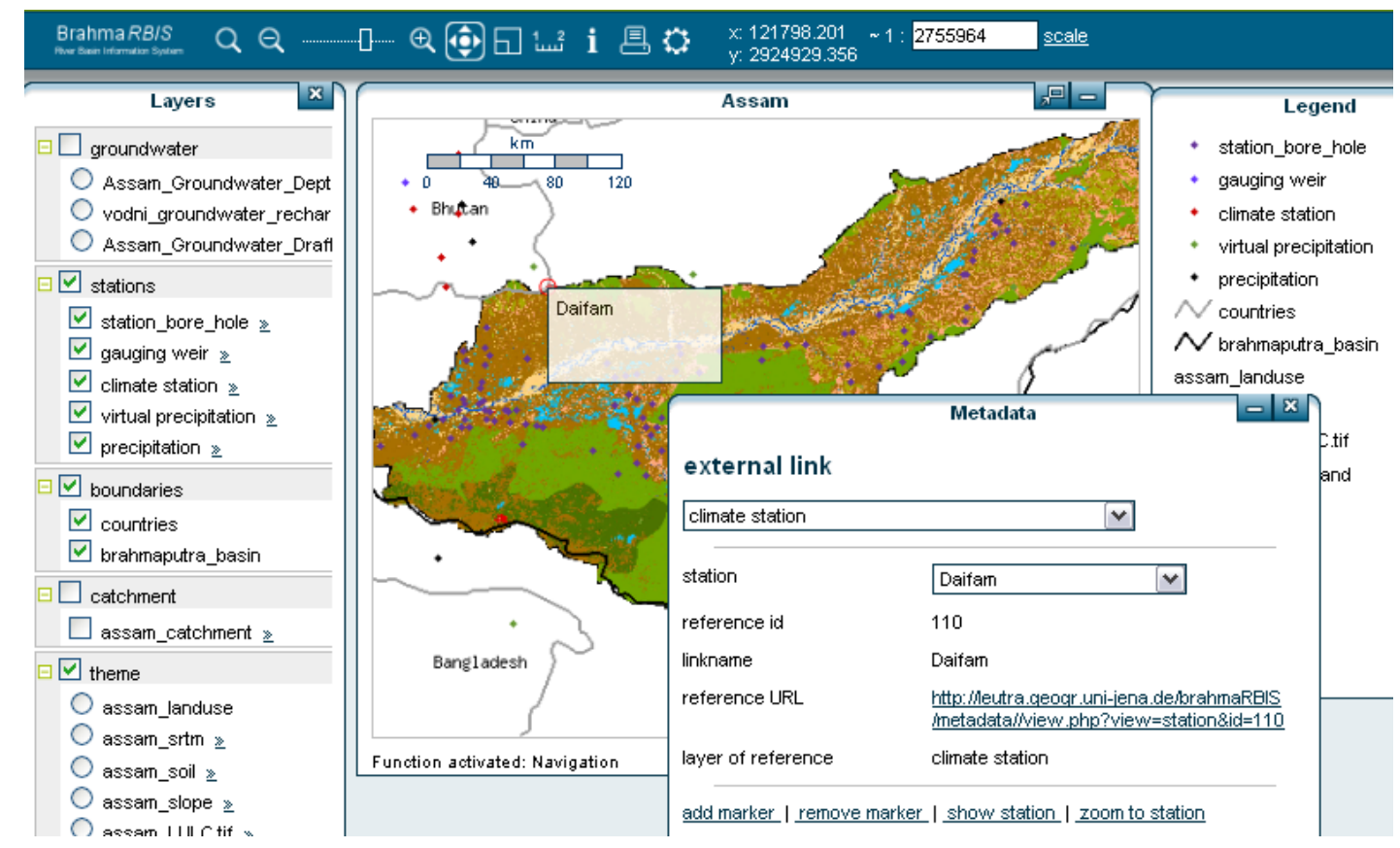

Figure 3. Linkage between station and station metadata and visualization of geodata in a map (source: BrahmaRBIS).

\subsection{RBIS core functions}

The system is based on a set of core functionalities offered to all RBIS modules. The most important are:

- User and permission management: All access to RBIS data is controlled by a fine-grained user and permission management. It is based on permission groups, actions (view, edit, delete) and data types (e.g. time series data or geo-data).

- Logging: Information about data manipulation activities (activity, new data, time, IP and user) and occurred errors are logged in order to ensure transparency, simplify the processing of support requests and allow the generation of activity reports.

- Help system: An online help system gives detailed information about the meaning of dataset attributes in RBIS. This also includes information about formats of the corresponding field or a list and description of all possible attributes in a given selection list.

- Template system: All RBIS forms can be saved as a template for later use. This significantly reduces the effort to add and upload new data, especially if datasets differ only slightly.

- Search via map: All spatially referenced datasets can be automatically displayed on a map. For example a climate station can be displayed in the climate stations layer on a map (Figure 3). A reversed search starting at a certain point, polygon or layer in a map is also possible and leads the user directly to the associated metadata and dataset.

- Search via attribute values: The user can search via the attribute values of each data type. 


\section{RBIS APPLICATION}

More than 15 RBIS instances have already been created in the context of EC or national funded projects (e.g. BMBF). Examples are:

- BrahmaRBIS and DanubeRBIS: environmental data from the Brahmaputra and Upper Danube river basins collected within the EC project BRAHMATWINN (Twinning European and South Asian river basins to enhance capacity and implement adaptive integrated water resources management approaches http://www.brahmatwinn.uni-jena.de).

- OBIS: the Okavango Basin Information System stores environmental information from the Okavango basin for the BMBF-funded project The Future Okavango (http://www.future-okavango.org).

- Vu Gia Thu Bon RBIS: environmental data from the Vu Gia Thu Bon River Basin from the BMBFfunded LUCCI project (Land Use and Climate Change interactions in the Vu Gia Thu Bon River Basin/Central Vietnam - http://www.lucci-vietnam.info).

In addition to be used as a mere data management system, RBIS is well suited as a data exchange platform for tools typically used in the context of integrated environmental management, e.g. remote sensing or environmental modelling. The following section will give an overview of the Integrated Landscape Management System and RBIS' role as its central data management component.

\subsection{Integrated Landscape Management System (ILMS)}

The Integrated Landscape Management System (ILMS) was developed at the Department of Geoinformatics, Hydrology and Modelling at the Friedrich Schiller University of Jena. It provides an integrated modular software platform and covers different steps of environmental systems analysis and planning in a flexible and user-friendly workflow (http://ilms.uni-jena.de, see Figure 4).

ILMS includes the following integrated components:

- ILMSinfo: the River Basin Information System (RBIS) for the central data management, analysis, visualization and presentation of different types of data.

- ILMSimage: a software for the identification and classification of real-world objects from satellite imagery using methods of object based image analysis (Matejka et al., 2008).

- ILMSgis: a software (GRASS-HRU) for the derivation of modelling entities using a Web Processing Service based on GRASS GIS and following the Hydrological Response Unit (HRU) approach (Schwartze, 2008, 2011).

- ILMSmodel: an environmental modelling framework (Jena Adaptable Modelling System - JAMS) for building, running and analyzing environmental simulation models (http://jams.uni-jena.de), e.g. hydrological models, (Kralisch et al., 2006).

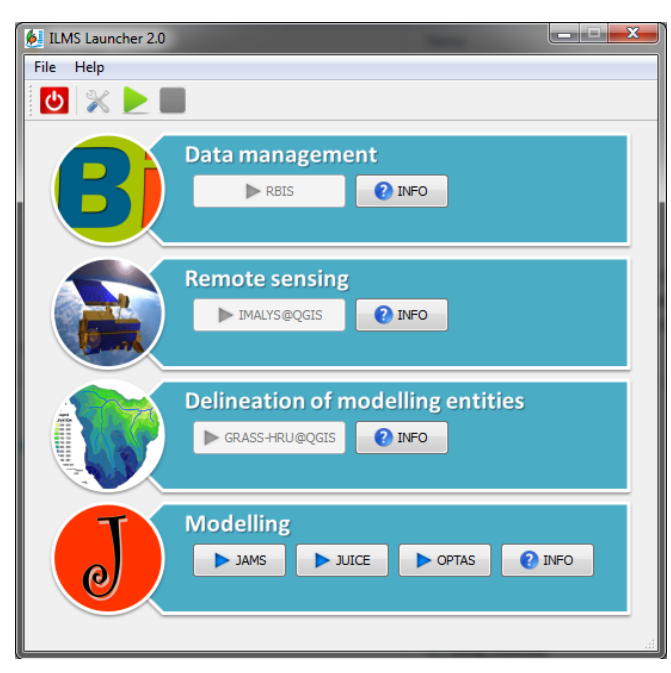

Figure 4. ILMS Launcher.

\subsection{Data management and exchange in ILMS}

All ILMS tools are able to retrieve most of their needed input data directly from RBIS. The produced output data can be stored and described by detailed metadata in RBIS. Output data of ILMSimage can be, for example, a land use classification which is also input data for the derivation of spatial modelling entities with ILMSgis. Derived modelling entities and gap filled time series data in turn are used as input for the ILMSmodel. The results of a simulation model, i.e. time series data, parameter sets, or maps data, can be directly uploaded to ILMSinfo (Zander et al., 2011).

For this reason RBIS is more than just a platform for data sharing. It can also serve as a platform providing well-presented and meaningful information for decision makers. Error! Reference source not found. shows the described exemplary data flow and data exchanges between ILMSgis, ILMSimage, ILMSmodel using ILMSinfo (RBIS) as central data store. 


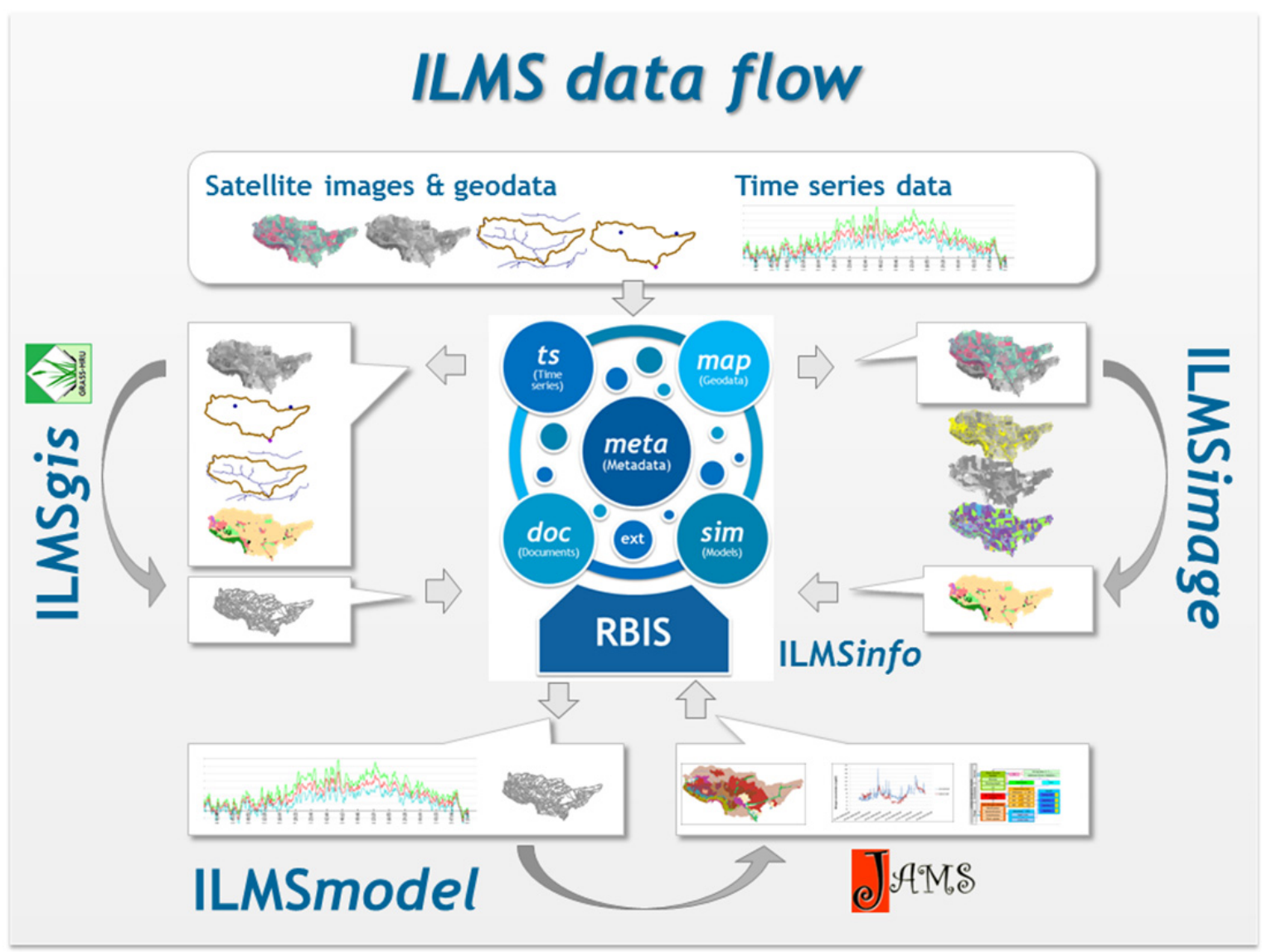

Figure 5. Data flow in ILMS showing the data flow from original data via data processing (ILMSgis and ILMSimage) to environmental simulation and modelling (ILMSmodel).

\section{SUMMARY AND OUTLOOK}

Due to its modular structure and interfaces RBIS is a flexible environmental information system that can be used in a wide range of applications related to environmental management. It provides support for sophisticated data management and data sharing in multidisciplinary research. To make sure that information is not distorted or lost during several processing steps, detailed metadata is recorded especially in the field of time series data management. The example application within ILMS also demonstrates its capability to serve as a central software component in more complex environmental management systems by providing core data exchange services, allowing related tools and applications to easily access the stored data and metadata.

To enhance the flexibility and open RBIS as a central data management system to an even broader range of applications, the ongoing RBIS development is especially targeted towards the further implementation of open standards and interfaces. In addition to the ISO19115 Standard for Geodata and WMS/WFS services being already part of RBIS, current activities include the implementation of the OGC Web Catalog Service (CSW) and the Sensor Observation Service (SOS)(http://www.opengeospatial.org/standards/sos), using WaterML2.0 as the time series encoding standard.

\section{ACKNOWLEDGMENTS}

The authors aknowledge the support of the German Federal Ministry of Education and Research which has funded the RBIS development as part of the InnoProfile program (project number: 03IP514). 
Zander et al., Environmental data management with the River Basin Information System

\section{REFERENCES}

ISO, INTERNATIONAL STANDARDS ORGANIZATION (2003): International Standard ISO 19115 Geographic information - Metadata. Reference Number ISO19115:2003(E).

Kralisch, S., P. Krause (2006). JAMS - A Framework for Natural Resource Model Development and Application, in Proceedings of the iEMSs Third Biannual Meeting "Summit on Environmental Modelling and Software", edited by A. Voinov, A. Jakeman, and A. Rizzoli, Burlington, USA.

Kralisch, S., F. Zander, P. Krause (2009). Coupling the RBIS Environmental Information System and the JAMS Modelling Framework. In: Proceedings of the 18th World IMACS Congress and MODSIM09 International Congress on Modelling and Simulation, edited by R. Anderssen, R. Braddock, and L. Newham, pp. 902-908, Cairns, Australia.

Matejka, E., M. Reinhold, P. Selsam (2008). IMALYS - an automated and database-integrated object-oriented classification system. In: GEOBIA 2008 - Pixels, Objects, Intelligence: Geographic Object Based Image Analysis for the 21 st Century, Proceedings GEOBIA 2008, Calgary, Kanada.

Schwartze, C. (2008). Deriving Hydrological Response Units (HRUs) using a Web Processing Service implementation based on GRASS GIS, Geoinformatics FCE CTU 2008, Workshop Proceedings Vol. 3, Prag.

Schwartze, C. (2011). Geospatial Virtual Appliances Using Open Source Software. In: Environmental Software Systems. Frameworks of eEnvironment. 9th IFIP WG 5.11 International Symposium, ISESS 2011, edited by Jiři Hřebíček, pp. 349-356, Brno, CZE.

Zander, F., Kralisch S., Busch C. and W.-A. Flügel (2011). RBIS - An Environmental Information System for Integrated Landscape Management. In: Environmental Software Systems. Frameworks of eEnvironment. 9th IFIP WG 5.11 International Symposium, ISESS 2011, edited by Jiř́i Hřebíček, pp. 349-356, Brno, CZE. 